Western Washington University Western CEDAR

2013

\title{
Anisotropic Classes of Inhomogeneous Pseudodifferential Symbols
}

Árpád Bényi

Western Washington University, arpad.benyi@wwu.edu

Marcin Bownik

University of Oregon

Follow this and additional works at: https://cedar.wwu.edu/math_facpubs

Part of the Mathematics Commons

\section{Recommended Citation}

Bényi, Árpád and Bownik, Marcin, "Anisotropic Classes of Inhomogeneous Pseudodifferential Symbols" (2013). Mathematics. 45. https://cedar.wwu.edu/math_facpubs/45

This Article is brought to you for free and open access by the College of Science and Engineering at Western CEDAR. It has been accepted for inclusion in Mathematics by an authorized administrator of Western CEDAR. For more information, please contact westerncedar@wwu.edu. 


\title{
ANISOTROPIC CLASSES OF INHOMOGENEOUS PSEUDODIFFERENTIAL SYMBOLS
}

\author{
ÁRPÁD BÉNYI AND MARCIN BOWNIK
}

\begin{abstract}
We introduce a class of pseudodifferential operators in the anisotropic setting induced by an expansive dilation $A$ which generalizes the classical isotropic class $S_{\gamma, \delta}^{m}$ of inhomogeneous symbols. We extend a well-known $L^{2}$-boundedness result to the anisotropic class $S_{\delta, \delta}^{0}(A), 0 \leq \delta<1$. As a consequence, we deduce that operators with symbols in the anisotropic class $S_{1,0}^{0}(A)$ are bounded on $L^{p}$ spaces, $1<p<\infty$.
\end{abstract}

\section{IntRoduCtion: DEFINITIONS AND STATEMENT OF MAIN RESUlT}

The study of pseudodifferential operators draws lots of its motivation from its applicability to approximate inverses or regularity of solutions in partial differential equations. A systematic study of these operators led to the introduction of the classical (isotropic, inhomogeneous) classes of symbols $S_{\gamma, \delta}^{m}$ and their tightly connected homogeneous counterparts denoted by $\dot{S}_{\gamma, \delta}^{m}$. For example, as it is well known, the characteristic polynomial of a partial differential operator of order $m$ and with constant coefficients represents a symbol in the class $S_{1,0}^{m}$. The adjective isotropic we use here points out that the spatial and frequency variables of the symbol have the same homogeneity. However, in several examples (such as the heat operator) there exists another natural scaling (such as parabolic) and thus we fall in the realm of anisotropic symbols. In our previous paper [1] we were interested in the study of multiplier, and more generally, pseudodifferential operators, with anisotropic homogeneous symbols $\dot{S}_{\gamma, \delta}^{m}(A)$.

This paper is a natural continuation of the investigations initiated in [1] for the general anisotropic setting. We will mainly concern ourselves with inhomogeneous symbols in the classes $S_{\gamma, \delta}^{0}(A)$, in particular with the extension of a classical boundedness result for anisotropic pseudodifferential operators with symbols $S_{\delta, \delta}^{0}(A), 0 \leq$ $\delta<1$. This result, in turn, implies the $L^{p}$-boundedness of the smaller class $S_{1, \delta}^{0}(A)$, $0 \leq \delta<1$. An example of symbol belonging to $S_{1,0}^{0}(A)$, for an appropriately chosen matrix $A$, is presented in detail in Example 3.1.

Let us now briefly recall the notation, the definition, and some of the results about the anisotropic homogeneous classes of pseudodifferential symbols. We follow the notation in Bownik's monograph [2]; see also [3], [4]. Given an expansive matrix A,

Date: October 7, 2011.

2010 Mathematics Subject Classification. Primary: 47G30, 42B20; Secondary: 42B15, 42B35.

Key words and phrases. Pseudodifferential operators, anisotropic inhomogeneous symbols, Calderón-Zygmund operators, anisotropic elementary symbols. 
that is a matrix for which all its eigenvalues $\lambda$ satisfy $|\lambda|>1$, we can first define a canonical quasi-norm $\rho_{A}$ associated to it. Specifically, if we let $P$ be some nondegenerate $n \times n$ matrix, and $|\cdot|$ the standard norm of $\mathbb{R}^{n}$, there exists an ellipsoid $\Delta=\left\{x \in \mathbb{R}^{n}:|P x|<1\right\}$ such that $|\Delta|=1$ and for some $r>1, \Delta \subset r \Delta \subset A \Delta$. Then, we can define a family of balls around the origin $B_{k}=A^{k} \Delta, k \in \mathbb{Z}$, that satisfy

$$
B_{k} \subset r B_{k} \subset B_{k+1} \text { and }\left|B_{k}\right|=b^{k},
$$

where $b=|\operatorname{det} A|$. The step homogeneous quasi-norm induced by $A$ is defined by

$$
\rho(x)=b^{j}, x \in B_{j+1} \backslash B_{j} \text {, and } \rho(0)=0 .
$$

It is straightforward to verify that $\rho$ satisfies a triangle inequality up to a constant and the homogeneity condition $\rho(A x)=b \rho(x), x \in \mathbb{R}^{n}$. Since any two homogeneous quasi-norms associated to a dilation $A$ are equivalent, we can talk about a canonical quasi-norm associated to $A$, which will be denoted by $\rho_{A}$. Similarly, we shall also consider a family of dilated balls $B_{k}^{*}, k \in \mathbb{Z}$, and a canonical quasi-norm $\rho_{A^{*}}$ associated to the adjoint (or transpose) matrix $A^{*}$. We are now ready to state the definition of anisotropic inhomogeneous symbols which is a natural modification of the homogeneous one, see [1].

Definition 1.1. We say that a symbol $\sigma(x, \xi)$ belongs to the anisotropic inhomogeneous class $S_{\gamma, \delta}^{m}(A)$ if it satisfies the estimates

$$
\left|\partial_{x}^{\alpha} \partial_{\xi}^{\beta}\left[\sigma\left(A^{-k_{1}} \cdot,\left(A^{*}\right)^{k_{2}} \cdot\right)\right]\left(A^{k_{1}} x,\left(A^{*}\right)^{-k_{2}} \xi\right)\right| \leq C_{\alpha, \beta}\left(1+\rho_{A^{*}}(\xi)\right)^{m},
$$

for all multi-indices $\alpha, \beta$ and $(x, \xi) \in \mathbb{R}^{n} \times \mathbb{R}^{n}$. Here, $k_{1}, k_{2} \in \mathbb{N}_{0}$ are given by

$$
k_{1}=\lfloor k \delta\rfloor, \quad k_{2}=\lfloor k \gamma\rfloor,
$$

where $k \in \mathbb{N}_{0}$ is such that $1+\rho_{A^{*}}(\xi) \sim|\operatorname{det} A|^{k}$, and $\lfloor\cdot\rfloor$ denotes the floor function.

The derivatives above should be interpreted as

$$
\partial_{x}^{\alpha} \partial_{\xi}^{\beta} \tilde{\sigma}\left(A^{k_{1}} x,\left(A^{*}\right)^{-k_{2}} \xi\right),
$$

where

$$
\tilde{\sigma}(x, \xi)=\sigma\left(A^{-k_{1}} x,\left(A^{*}\right)^{k_{2}} \xi\right),
$$

and $k_{1}, k_{2} \in \mathbb{N}_{0}$ are as in (1.2). The notation $\sim$ has the following interpretation: we pick $k$ to be the unique non-negative integer such that the frequency variable $\xi$ belongs to the annulus $B_{k+1}^{*} \backslash B_{k}^{*}$ if $k>0$, or the ball $B_{1}^{*}$ if $k=0$. Consequently, we require estimates on the derivatives of a symbol $\sigma$ that hold uniformly after appropriate rescaling depending on the location of the frequency variable $\xi$. Recall from [1] that in the homogeneous variant $\dot{S}_{\gamma, \delta}^{m}(A)$ of Definition 1.1, $k$ is the unique integer such that $0 \neq \xi \in B_{k+1}^{*} \backslash B_{k}^{*}$, and hence $k$ can take negative values as well.

As explained in [1], Definition 1.1 recovers not only the well-known isotropic classes $S_{\gamma, \delta}^{m}=S_{\gamma, \delta}^{m}\left(2 I_{n}\right)$, but also the so-called anisotropic classes $S_{a ; \gamma, \delta}^{m}$ previously investigated in the works of Leopold [14] and Garello [9]. Moreover, the generality of our definition is very useful when dealing with a general, non-diagonal anisotropy. Furthermore, we proved in the general anisotropic setting that the class $\dot{S}_{1,1}^{0}(A)$ corresponds to operators with Calderón-Zygmund kernels which are bounded on 
anisotropic Triebel-Lizorkin and Besov spaces. In particular, we recovered the results of Grafakos-Torres [10] for the homogeneous isotropic class $\dot{S}_{1,1}^{m}$; see [1].

We remark right away that Definition 1.1 implies that the anisotropic CalderónVaillancourt class $S_{0,0}^{0}(A)$ is independent of the matrix $A$, and, as such, it coincides with the isotropic version $S_{0,0}^{0}$. Therefore, we conclude that $S_{0,0}^{0}(A)$ is bounded (in general, only) on $L^{2}$. This is a well known result of Calderón-Vaillancourt [6]; see also Stein's monograph [16, Section VII.2.4]. By contrast, the $L^{2}$ boundedness of the class $S_{1,1}^{0}(A)$ fails. A standard counterexample of a pseudodifferential symbol in the (isotropic) class $S_{1,1}^{0}$ that yields unbounded operator on $L^{2}$ can be found in [16, Section VII.1.2]. This example can be extended to the generic anisotropic setting, see Example 3.2.

In analogy with the isotropic setting, the Schwarz kernel of an operator with symbol in $S_{1,1}^{0}(A)$ is Calderón-Zygmund, and thus $L^{p}$ boundedness fails for all $1<p<$ $\infty$. The computations that verify the anisotropic Calderón-Zygmund estimates are left to the interested reader. They are essentially the same as the ones detailed in [1, Theorem 4.3] for the homogeneous class $\dot{S}_{1,1}^{0}(A)$ with the obvious modifications implied by using the inhomogeneous Littlewood-Paley decomposition (2.2).

The comments above beg a natural question: what happens for the symbols in $S_{\delta, \delta}^{0}(A)$ ? Recall that, by Definition 1.1, a symbol $\sigma \in S_{\delta, \delta}^{0}(A)$ satisfies the following inequalities:

$$
\left|\partial_{x}^{\alpha} \partial_{\xi}^{\beta}\left[\sigma\left(A^{-k^{\prime}} \cdot,\left(A^{*}\right)^{k^{\prime}} \cdot\right)\right]\left(A^{k^{\prime}} x,\left(A^{*}\right)^{-k^{\prime}} \xi\right)\right| \leq C_{\alpha, \beta},
$$

for all multi-indices $\alpha, \beta,(x, \xi) \in \mathbb{R}^{n} \times \mathbb{R}^{n}$, and $k \in \mathbb{N}_{0}$ such that $1+\rho_{A^{*}}(\xi) \sim|\operatorname{det} A|^{k}$, where $k^{\prime}=\lfloor k \delta\rfloor$. Associated to such a symbol $\sigma(x, \xi)$, we have a pseudodifferential operator, a priori defined on $\mathcal{S}$ :

$$
T_{\sigma} f(x)=\sigma(x, D) f(x)=\int_{\mathbb{R}^{n}} \sigma(x, \xi) \widehat{f}(\xi) e^{i x \cdot \xi} d \xi .
$$

Our main result is the anisotropic extension of the following well known boundedness result for the isotropic class $S_{\delta, \delta}^{0}$.

Theorem 1.1. Let $\sigma \in S_{\delta, \delta}^{0}(A)$ for some $0 \leq \delta<1$. Then, the pseudodifferential operator $T_{\sigma}$ extends to a bounded operator on $L^{2}\left(\mathbb{R}^{n}\right)$.

The inhomogeneous classes of symbols are nested: $S_{\gamma_{1}, \delta_{1}}^{m_{1}}(A) \subset S_{\gamma_{2}, \delta_{2}}^{m_{2}}(A)$ if $m_{1} \leq$ $m_{2}, \gamma_{2} \leq \gamma_{1}$, and $\delta_{1} \leq \delta_{2}$. Thus, Theorem 1.1 also holds for the class $S_{\gamma, \delta}^{0}(A)$, where $0 \leq \delta \leq \gamma \leq 1$ and $\delta<1$. This condition on the indices defining the classes of symbols is known to be sharp in the isotropic setting, see the works of Hörmander [11] and Kumano-go [13].

Section 2 of our paper is devoted to the proof of Theorem 1.1. We divide this proof into several steps in which we explain our strategy leading to the conclusion we wish to achieve. Our approach is inspired by Stein's book [16, Theorem 2 in Section VII.2] albeit with some necessary changes reflecting a more complicated nature of symbols in anisotropic classes.

In Section 3 we give a couple of examples of anisotropic symbols. We also give an alternative proof of the boundedness of the class $S_{1,0}^{0}(A)$ using a reduction to 
elementary symbols. Our approach is guided by Coifman and Meyer's work [5] and the nice exposition in Journé's monograph [12].

\section{Proof of Theorem 1.1 Via Almost orthogonality}

We begin with the following elementary lemma.

Lemma 2.1. Suppose that $A$ is an expansive matrix, and let $\lambda_{-}=\min _{\lambda \in \sigma(A)}|\lambda|$. Let $\zeta_{-}=\ln \lambda_{-} / \ln b$, where $b=|\operatorname{det} A|$. Finally, suppose that $N>1 /\left(2 \zeta_{-}\right)$. Then, there exists some $C>0$ such that for all $j \in \mathbb{Z}$ we have

$$
\int_{\mathbb{R}^{n}}\left(1+\left|A^{j} z\right|^{2}\right)^{-N} d z \leq C b^{-j}
$$

Proof. Let $2 N>1 / \zeta>1 / \zeta_{-}$. By Lemma [2, Lemma 3.2], there exists a constant $c>0$ such that

$$
1+|x| \geq c\left(1+\rho_{A}(x)\right)^{\zeta_{-}} \quad \text { for all } x \in \mathbb{R}^{n} .
$$

Using the previous inequality and a change of variables, we get

$$
\begin{aligned}
\int_{\mathbb{R}^{n}}\left(1+\left|A^{j} z\right|^{2}\right)^{-N} d z & \leq C \int_{\mathbb{R}^{n}}\left(1+\rho_{A}\left(A^{j} z\right)\right)^{-2 N \zeta} d z \\
& \leq C b^{-j} \int_{\mathbb{R}^{n}}\left(1+\rho_{A}(z)\right)^{-2 N \zeta} d z \leq C b^{-j} .
\end{aligned}
$$

The last inequality is a consequence of $2 N \zeta>1$.

The proof of Theorem 1.1 is divided into several steps.

Step 1. First, we perform a reduction to symbols $\sigma$ with compact support in $\mathbb{R}^{n} \times \mathbb{R}^{n}$. Take $\phi$ a fixed smooth function with compact support with $\phi(0,0)=1$. For each $j \in \mathbb{N}$ define

$$
\sigma_{j}(x, \xi)=\sigma(x, \xi) \phi\left(A^{-j} x,\left(A^{*}\right)^{-j} \xi\right) .
$$

Using support considerations and the chain rule, we can show that the symbols $\sigma_{j} \in S_{\delta, \delta}^{0}(A)$ uniformly for $j \in \mathbb{N}$. Furthermore, for all $f \in \mathcal{S}$,

$$
T_{\sigma_{j}} f \rightarrow T_{\sigma} f \quad \text { as } j \rightarrow \infty,
$$

in the topology of $\mathcal{S}$, see [16, Section VI.1.3]. The reduction to symbols with compact support will allows us to automatically justify all operations appearing below such as integration by parts. The explicit dependence on $j$ will be suppressed and all of our estimates will be independent of $j$. In the rest of the proof we shall simply assume that $\sigma \in S_{\delta, \delta}^{0}(A)$ has compact support.

Step 2. We decompose now the operator $T_{\sigma}$ in the frequency domain as

$$
T_{\sigma}=\sum_{j=0}^{\infty} T_{\sigma_{j}}, \quad \text { where } \sigma_{j}(x, \xi)= \begin{cases}\sigma(x, \xi) \psi\left(\left(A^{*}\right)^{-j} \xi\right), & j \geq 0, \\ \sigma(x, \xi) \varphi(\xi), & j=0 .\end{cases}
$$

Here, $\varphi, \psi \in \mathcal{S}$ satisfy $\operatorname{supp} \varphi \subset B_{1}^{*}, \operatorname{supp} \psi \subset B_{1}^{*} \backslash B_{-1}^{*}$ and

$$
\varphi(\xi)+\sum_{j=1}^{\infty} \psi\left(\left(A^{*}\right)^{-j} \xi\right)=1 \quad \text { for all } \xi \in \mathbb{R} .
$$


As in Step 1, one can show that the symbols $\sigma_{j}$ are uniformly in $S_{\delta, \delta}^{0}(A)$.

Step 3. We establish that the operators $T_{\sigma_{j}}$ are very close to being mutually orthogonal. Fix a sufficiently large $\omega \in \mathbb{N}$, which will be determined in the next step. We break the sum (2.1) into a finite sum of infinite series

$$
T_{\sigma}=\sum_{r=1}^{\omega}\left(\sum_{\substack{j=0 \\ j \equiv r}}^{\infty} T_{\sigma_{j}}\right) .
$$

It suffices to prove the boundedness of each series separately corresponding to some fixed $r=1, \ldots, \omega$.

Observe that $T_{\sigma_{j}}=T_{\sigma} \Delta_{j}$, where $\Delta_{j}$ is the multiplier operator given by

$$
\widehat{\left(\Delta_{j} f\right)}(\xi)= \begin{cases}\hat{f}(\xi) \psi\left(\left(A^{*}\right)^{-j} \xi\right), & j \geq 0 \\ \hat{f}(\xi) \varphi(\xi), & j=0 .\end{cases}
$$

By the support condition on $\psi$ we have that

$$
T_{\sigma_{j}}\left(T_{\sigma_{k}}\right)^{*}=T_{\sigma} \Delta_{j}\left(\Delta_{k}\right)^{*}\left(T_{\sigma}\right)^{*}=0 \quad \text { for }|j-k| \geq 2 .
$$

Step 4. This is the key part where we estimate the kernel of $\left(T_{\sigma_{j}}\right)^{*} T_{\sigma_{k}}$. By a direct calculation, as in the proof of [16, Theorem 2 in VII.2.5], we have

$$
\left(T_{\sigma_{j}}\right)^{*} T_{\sigma_{k}} f(x)=\int_{\mathbb{R}^{n}} K(x, y) f(y) d y,
$$

where the kernel

$$
K(x, y)=\int_{\mathbb{R}^{n} \times \mathbb{R}^{n} \times \mathbb{R}^{n}} \overline{\sigma_{k}}(z, \eta) \sigma_{j}(z, \xi) e^{i[\xi \cdot(z-y)-\eta \cdot(z-x)]} d z d \eta d \xi
$$

We will estimate the kernel $K$ by exploiting the oscillatory nature of the exponential and the relative smoothness of symbols $\overline{\sigma_{k}}$ and $\sigma_{j}$. This will be achieved by integration by parts in all three variables $z, \eta, \xi$. Unlike the isotropic setting, we need to change variables first. This is necessary since the anisotropic condition (1.3) involves derivatives of a dilated symbol.

Assume that $j<k$ belong to the same sum as in Step 3, i.e., $j \equiv k \bmod \omega$. Define $j^{\prime}=\lfloor j \delta\rfloor, k^{\prime}=\lfloor k \delta\rfloor$. By a change of variables in (2.3) we have

$$
\begin{array}{r}
K\left(A^{-k^{\prime}} x, A^{-k^{\prime}} y\right)=b^{j^{\prime}} \int_{\mathbb{R}^{n} \times \mathbb{R}^{n} \times \mathbb{R}^{n}} \overline{\sigma_{k}}\left(A^{-k^{\prime}} z,\left(A^{*}\right)^{k^{\prime}} \eta\right) \sigma_{j}\left(A^{-k^{\prime}} z,\left(A^{*}\right)^{j^{\prime}} \xi\right) \\
e^{i\left[\left(A^{*}\right)^{j^{\prime}-k^{\prime}} \xi \cdot(z-y)-\eta \cdot(z-x)\right]} d z d \eta d \xi
\end{array}
$$

Indeed, this is a consequence of the changes of variables $z \mapsto A^{-k^{\prime}} z, \eta \mapsto\left(A^{*}\right)^{k^{\prime}} \eta$, $\xi \mapsto\left(A^{*}\right)^{j^{\prime}} \xi$ and using the identity

$$
\left(A^{*}\right)^{j^{\prime}} \xi \cdot\left(A^{-k^{\prime}} z-A^{-k^{\prime}} y\right)-\left(A^{*}\right)^{k^{\prime}} \eta \cdot\left(A^{-k^{\prime}} z-A^{-k^{\prime}} x\right)=\left(A^{*}\right)^{j^{\prime}-k^{\prime}} \xi \cdot(z-y)-\eta \cdot(z-x) .
$$

Now set

$$
\tilde{\sigma}_{k}(z, \eta)=\sigma_{k}\left(A^{-k^{\prime}} z,\left(A^{*}\right)^{k^{\prime}} \eta\right), \quad \tilde{\sigma}_{j}(z, \xi)=\sigma_{j}\left(A^{-k^{\prime}} z,\left(A^{*}\right)^{j^{\prime}} \xi\right) .
$$


By support considerations we have

$$
\begin{aligned}
& \tilde{\sigma}_{k}(z, \eta) \neq 0 \Longrightarrow \rho_{A^{*}}(\eta) \sim b^{k(1-\delta)}, \\
& \tilde{\sigma}_{j}(z, \xi) \neq 0 \Longrightarrow \rho_{A^{*}}(\xi) \sim b^{j(1-\delta)} .
\end{aligned}
$$

Now, since $\operatorname{supp} \sigma_{k} \subset \mathbb{R}^{n} \times\left(B_{k+1}^{*} \backslash B_{k-1}^{*}\right)$, the condition (1.3) satisfied by $\sigma_{k}$ reduces to

$$
\left|\partial_{z}^{\alpha} \partial_{\eta}^{\beta}\left[\sigma_{k}\left(A^{-k^{\prime}} \cdot,\left(A^{*}\right)^{k^{\prime}} \cdot\right)\right]\left(A^{k^{\prime}} x,\left(A^{*}\right)^{-k^{\prime}} \eta\right)\right| \leq C_{\alpha, \beta} \quad \text { for all multi-indices } \alpha, \beta,
$$

and for all $(z, \eta) \in \mathbb{R}^{n} \times \mathbb{R}^{n}$. This is due to the fact that only one dilate of $\sigma_{k}$, that is $\tilde{\sigma}_{k}$, can give a non-zero contribution in (1.3). Thus, we have

$$
\left\|\partial_{z}^{\alpha} \partial_{\eta}^{\beta} \tilde{\sigma}_{k}\right\|_{\infty} \leq C_{\alpha, \beta} \quad \text { for all multi-indices } \alpha, \beta \text {. }
$$

In the same way we can deduce that

$$
\left|\partial_{z}^{\alpha} \partial_{\xi}^{\beta}\left[\sigma_{j}\left(A^{-j^{\prime}} \cdot,\left(A^{*}\right)^{j^{\prime}} \cdot\right)\right]\left(A^{j^{\prime}} z,\left(A^{*}\right)^{-j^{\prime}} \xi\right)\right| \leq C_{\alpha, \beta} \quad \text { for all multi-indices } \alpha, \beta,
$$

and for all $(z, \xi) \in \mathbb{R}^{n} \times \mathbb{R}^{n}$. Thus, using the chain rule and recalling that $j^{\prime} \leq k^{\prime}$, we have

$$
\left|\partial_{z}^{\alpha} \partial_{\xi}^{\beta} \tilde{\sigma}_{j}(z, \xi)\right| \leq C|| A^{j^{\prime}-k^{\prime}}||^{|\alpha|}\left|\partial_{z}^{\alpha} \partial_{\xi}^{\beta}\left[\sigma_{j}\left(A^{-j^{\prime}} \cdot,\left(A^{*}\right)^{j^{\prime}} \cdot\right)\right]\left(A^{j^{\prime}-k^{\prime}} z, \xi\right)\right| \leq C_{\alpha, \beta} .
$$

This shows that

$$
\left\|\partial_{z}^{\alpha} \partial_{\xi}^{\beta} \tilde{\sigma}_{j}\right\|_{\infty} \leq C_{\alpha, \beta} \quad \text { for all multi-indices } \alpha, \beta .
$$

Since

$$
\frac{\left(I-\Delta_{z}\right)^{N}}{\left(1+\left|\left(A^{*}\right)^{j^{\prime}-k^{\prime}} \xi-\eta\right|^{2}\right)^{N}} e^{i\left(\left(A^{*}\right)^{j^{\prime}-k^{\prime}} \xi-\eta\right) \cdot z}=e^{i\left(\left(A^{*}\right)^{j^{\prime}-k^{\prime}} \xi-\eta\right) \cdot z},
$$

integrating by parts in the $z$ variable in (2.4) yields

$$
\begin{aligned}
K\left(A^{-k^{\prime}} x, A^{-k^{\prime}} y\right)=b^{j^{\prime}} \int_{\mathbb{R}^{n} \times \mathbb{R}^{n} \times \mathbb{R}^{n}}\left(I-\Delta_{z}\right)^{N}\left[\overline{\tilde{\sigma}_{k}}(z, \eta) \tilde{\sigma}_{j}(z, \xi)\right] & \frac{e^{i\left[\left(A^{*}\right)^{j^{\prime}-k^{\prime}} \xi \cdot(z-y)-\eta \cdot(z-x)\right]}}{\left(1+\left|\left(A^{*}\right)^{j^{\prime}-k^{\prime}} \xi-\eta\right|^{2}\right)^{N}} d z d \eta d \xi .
\end{aligned}
$$

Next, we integrate by parts in (2.8) with respect to the $\xi$ and $\eta$ variables, respectively. Similarly to our computation above, we use

$$
\frac{\left(I-\Delta_{\eta}\right)^{N}}{\left(1+|x-z|^{2}\right)^{N}} e^{-i \eta \cdot(x-z)}=e^{-i \eta \cdot(x-z)},
$$

and

$$
\frac{\left(I-\Delta_{\xi}\right)^{N}}{\left(1+\left|A^{j^{\prime}-k^{\prime}}(z-y)\right|^{2}\right)^{N}} e^{i\left(A^{*}\right)^{j^{\prime}-k^{\prime}} \xi \cdot(z-y)}=e^{i\left(A^{*}\right)^{j^{\prime}-k^{\prime}} \xi \cdot(z-y)} .
$$

The end result of these integrations by parts is the identity

$$
\begin{aligned}
K\left(A^{-k^{\prime}} x, A^{-k^{\prime}} y\right) & =b^{j^{\prime}} \int_{\mathbb{R}^{n} \times \mathbb{R}^{n} \times \mathbb{R}^{n}}\left[\frac{\left(I-\Delta_{\xi}\right)^{N}}{\left(1+\left|A^{j^{\prime}-k^{\prime}}(z-y)\right|^{2}\right)^{N}} \frac{\left(I-\Delta_{\eta}\right)^{N}}{\left(1+|x-z|^{2}\right)^{N}}\right. \\
& \left.\frac{\left(I-\Delta_{z}\right)^{N}\left[\tilde{\sigma}_{k}(z, \eta) \tilde{\sigma}_{j}(z, \xi)\right]}{\left(1+\left|\left(A^{*}\right)^{j^{\prime}-k^{\prime}} \xi-\eta\right|^{2}\right)^{N}}\right] e^{i\left[\left(A^{*}\right)^{j^{\prime}-k^{\prime}} \xi \cdot(z-y)-\eta \cdot(z-x)\right]} d z d \eta d \xi .
\end{aligned}
$$


Since $j<k$ and $j \equiv k \bmod \omega$, we have $j+\omega \leq k$. Take any $\eta$ and $\xi$ as in (2.5). Thus, for some $c>0$,

$$
\rho_{A^{*}}(\eta) \geq c b^{(k-j)(1-\delta)} \rho_{A^{*}}\left(\left(A^{*}\right)^{j^{\prime}-k^{\prime}} \xi\right) .
$$

By the anisotropic version of the triangle inequality, there exists a constant $\omega_{0} \in \mathbb{N}$ such that

$$
\rho_{A^{*}}(\eta) \geq b^{\omega_{0}} \rho_{A^{*}}(\xi) \Longrightarrow b^{\omega_{0}} \rho_{A^{*}}(\eta) \geq \rho_{A^{*}}(\xi-\eta) \geq b^{-\omega_{0}} \rho_{A^{*}}(\eta)
$$

Hence, by choosing $\omega$ such that $c b^{\omega(1-\delta)}>b^{\omega_{0}}$ we have

$$
\rho_{A^{*}}\left(\left(A^{*}\right)^{j^{\prime}-k^{\prime}} \xi-\eta\right) \sim b^{k(1-\delta)}
$$

and

$$
\left|\left(A^{*}\right)^{j^{\prime}-k^{\prime}} \xi-\eta\right| \geq \rho_{A^{*}}\left(\left(A^{*}\right)^{j^{\prime}-k^{\prime}} \xi-\eta\right)^{\zeta_{-}} \sim b^{k(1-\delta) \zeta_{-}},
$$

where $\zeta_{-}=\ln \lambda_{-} / \ln b$. Therefore, whenever the expression $\left(1+\left|\left(A^{*}\right)^{j^{\prime}-k^{\prime}} \xi-\eta\right|^{2}\right)^{-N}$ is hit by derivatives in $\eta$ it will remain bounded by $C b^{k(1-\delta) \zeta_{-}}$. The same is true for derivatives in $\xi$ with the additional application of the chain rule and the fact that $j^{\prime}-k^{\prime}<0$.

Inserting the estimates (2.5), (2.6), (2.7), and (2.10) into (2.9) and integrating over variables $\xi$ and $\eta$ yields

$$
\left|K\left(A^{-k^{\prime}} x, A^{-k^{\prime}} y\right)\right| \leq C b^{j^{\prime}+(k+j)(1-\delta)-k(1-\delta) \zeta_{-} 2 N} \int_{\mathbb{R}^{n}} Q\left(A^{j^{\prime}-k^{\prime}}(z-y)\right) Q(x-z) d z,
$$

where $Q(v)=\left(1+|v|^{2}\right)^{-N}$. Thus,

$$
|K(x, y)| \leq C b^{j^{\prime}+(k+j)(1-\delta)-k(1-\delta) \zeta_{-} 2 N} \int_{\mathbb{R}^{n}} Q\left(A^{j^{\prime}-k^{\prime}} z-A^{j^{\prime}} y\right) Q\left(A^{k^{\prime}} x-z\right) d z .
$$

Step 5. Estimate (2.11) allows us to control both

$$
\int_{\mathbb{R}^{n}}|K(x, y)| d y \quad \text { and } \quad \int_{\mathbb{R}^{n}}|K(x, y)| d x .
$$

Indeed, by Lemma 2.1, we first obtain

$$
\begin{gathered}
\int_{\mathbb{R}^{n} \times \mathbb{R}^{n}} Q\left(A^{j^{\prime}-k^{\prime}} z-A^{j^{\prime}} y\right) Q\left(A^{k^{\prime}} x-z\right) d y d z \leq C b^{-j^{\prime}} \int_{\mathbb{R}^{n}} Q\left(A^{k^{\prime}} x-z\right) d z \leq C b^{-j^{\prime}} \\
\int_{\mathbb{R}^{n} \times \mathbb{R}^{n}} Q\left(A^{j^{\prime}-k^{\prime}} z-A^{j^{\prime}} y\right) Q\left(A^{k^{\prime}} x-z\right) d x d z \leq C b^{-k^{\prime}} \int_{\mathbb{R}^{n}} Q\left(A^{j^{\prime}-k^{\prime}} x-z\right) d z \\
\leq C b^{-k^{\prime}} b^{k^{\prime}-j^{\prime}}=C b^{-j^{\prime}} .
\end{gathered}
$$

Thus, by (2.11)

$$
\int_{\mathbb{R}^{n}}|K(x, y)| d y \leq C b^{(k+j)(1-\delta)-k(1-\delta) \zeta_{-} 2 N},
$$

and the same estimate holds for $\int_{\mathbb{R}^{n}}|K(x, y)| d x$. Therefore, Schur's lemma yields

$$
\left\|\left(T_{\sigma_{j}}\right)^{*} T_{\sigma_{k}}\right\| \leq C b^{(k+j)(1-\delta)-k(1-\delta) \zeta_{-} 2 N},
$$


for all $j \leq k+\omega$. Furthermore, by taking adjoints, we also have

$$
\left\|\left(T_{\sigma_{j}}\right)^{*} T_{\sigma_{k}}\right\| \leq C b^{2 \max (k, j)(1-\delta)\left(1-\zeta_{-} N\right)} \quad \text { for }|j-k| \geq \omega .
$$

Step 6. In this last step, we apply a "cruder" version of Cotlar's lemma, see [16, p. 282]. By Step 5, we have

$$
\left\|\left(T_{\sigma_{j}}\right)^{*} T_{\sigma_{k}}\right\| \leq \gamma(j) \gamma(k) \quad \text { for }|j-k| \geq \omega,
$$

where $\gamma(j)=C b^{-\epsilon j}$ and $\epsilon=(1-\delta)\left(\zeta_{-} N-1\right)>0$. Moreover, by Step 3, $T_{\sigma_{j}}\left(T_{\sigma_{k}}\right)^{*}=0$ for $|j-k| \geq 2$. Hence, it suffices to show that the operators $T_{\sigma_{k}}$ are uniformly bounded. By (2.6), the symbols $\tilde{\sigma}_{k}$ belong to $S_{0,0}^{0}(A)$ uniformly in $k$. Recall that the anisotropic class $S_{0,0}^{0}(A)$ coincides with its isotropic counterpart $S_{0,0}^{0}$. Therefore, the $L^{2}$ boundedness of the class $S_{0,0}^{0}$ implies that the pseudodifferential operators $T_{\tilde{\sigma}_{k}}$ are bounded uniformly in $k$. Moreover, by a dilation argument, we have

$$
T_{\sigma_{k}}=D_{A^{k}} T_{\tilde{\sigma}_{k}} D_{A^{-k}} .
$$

Here, $D_{A} f(x)=|\operatorname{det} A|^{1 / 2} f(A x)$ denotes the dilation operator, which is an isometry on $L^{2}\left(\mathbb{R}^{n}\right)$. Consequently, the operators $T_{\sigma_{k}}$ are bounded uniformly in $k$. By the above mentioned variant of Cotlar's lemma, each operator $\sum_{k \equiv r \bmod \omega} T_{\sigma_{k}}, r=1, \ldots, \omega$, is bounded on $L^{2}\left(\mathbb{R}^{n}\right)$. By Step 3, this completes the proof of Theorem 1.1.

Remark 2.1. We remark that the proof of Theorem 1.1 requires the estimates (1.3) on the derivatives $|\alpha|,|\beta| \leq N$ of a symbol in $S_{\delta, \delta}^{0}(A)$ up to the order $N>2 / \zeta_{-}$. Note that, for any dilation $A$, we always have $0<\zeta_{-} \leq 1 / n$. In the isotropic case, we have the equality $\zeta_{-}=1 / n$. Moreover, by the result of Coifman and Meyer [5], it is known that $N>n / 2$ is enough to guarantee the $L^{2}$ boundedness for pseudodifferential operators in the isotropic class $S_{\delta, \delta}^{0}, 0 \leq \delta<1$, and this is optimal. Hence, it is tempting to conjecture that Theorem 1.1 holds with a weaker requirement on the order of partial derivatives $N>1 /\left(2 \zeta_{-}\right)$.

\section{EXAMPLES AND ELEMENTARY SYMBOLS}

In this section we give another proof for the boundedness of symbols in so-called anisotropic Coifman-Meyer class $S_{1,0}^{0}(A)$. Because pseudodifferential operators with symbols $\sigma \in S_{1,0}^{0}(A)$ have (anisotropic) Calderón-Zygmund kernels, by Theorem 1.1 we also get that $\sigma(x, D)$ is bounded on all $L^{p}, 1<p<\infty$.

Theorem 3.1. Let $\sigma \in S_{1,0}^{0}(A)$. Then, $\sigma(x, D)$ extends to a bounded operator on $L^{p}$, $1<p<\infty$.

By Definition 1.1, a symbol $\sigma \in S_{1,0}^{0}(A)$ satisfies the following inequalities:

$$
\left|\partial_{x}^{\alpha} \partial_{\xi}^{\beta}\left[\sigma\left(\cdot,\left(A^{*}\right)^{k} \cdot\right)\right]\left(x,\left(A^{*}\right)^{-k} \xi\right)\right| \leq C_{\alpha, \beta},
$$

for all multi-indices $\alpha, \beta,(x, \xi) \in \mathbb{R}^{n} \times \mathbb{R}^{n}$, and $k \in \mathbb{N}$ such that $1+\rho_{A^{*}}(\xi) \sim b^{k}$. An example of symbol satisfying these inequalities can be obtained by an appropriate modification of [1, Example 2.1]. 
Example 3.1. Let $\varphi$ be an infinitely differentiable real valued function such that, for some constants $C_{1}, C_{2}, C_{3}>0, C_{1} \leq \varphi(x) \leq C_{2}$ and $\left|\varphi^{(k)}(x)\right| \leq C_{3}$ for all $x \in \mathbb{R}$ and all $k \geq 1$. For example, $\varphi(x)=2+\sin x$ satisfies these conditions. We consider the symbol $\sigma(x, \xi)$, with $x=\left(x_{1}, x_{2}\right), \xi=\left(\xi_{1}, \xi_{2}\right) \in \mathbb{R}^{2}$, defined by

$$
\sigma\left(\left(x_{1}, x_{2}\right),\left(\xi_{1}, \xi_{2}\right)\right)=\frac{\xi_{1}^{6}+\varphi\left(x_{2}\right) \xi_{2}^{6}}{1+\xi_{1}^{6}+\xi_{2}^{2}+\varphi\left(x_{2}\right) \xi_{2}^{6}} .
$$

We claim that $\sigma \in S_{1,0}^{0}(A)$, where $A$ is a $2 \times 2$ diagonal matrix with diagonal entries $\sqrt{2}, 2 \sqrt{2}$. More precisely, we claim to have estimates of the following form

$$
\left|\partial_{x_{1}}^{\beta_{1}} \partial_{x_{2}}^{\beta_{2}} \partial_{\xi_{1}}^{\alpha_{1}} \partial_{\xi_{2}}^{\alpha_{2}} \sigma\left(\left(x_{1}, x_{2}\right),\left(\xi_{1}, \xi_{2}\right)\right)\right| \lesssim\left(1+\rho_{A^{*}}\left(\xi_{1}, \xi_{2}\right)\right)^{-\left\|\left(\alpha_{1}, \alpha_{2}\right)\right\|} .
$$

where

$$
\rho_{A^{*}}\left(\xi_{1}, \xi_{2}\right)=\max _{i=1,2}\left(\left|\xi_{1}\right|^{2},\left|\xi_{2}\right|^{2 / 3}\right),\left\|\left(\alpha_{1}, \alpha_{2}\right)\right\|=\frac{1}{2} \alpha_{1}+\frac{3}{2} \alpha_{2} .
$$

We shall verify directly these estimates only for $(1,0,0,0)$ and its permutations. We will always break down our analysis depending on the relative size of $\left|\xi_{1}\right|^{3}$ and $\left|\xi_{2}\right|$ (which determine the quasi-norm).

1. $(\beta, \alpha)=(1,0,0,0)$. This case is trivial, the derivative being zero.

2. $(\beta, \alpha)=(0,1,0,0)$. We compute

$$
\partial_{x_{2}} \sigma(x, \xi)=\frac{\varphi^{\prime}\left(x_{2}\right) \xi_{2}^{6}\left(1+\xi_{2}^{2}\right)}{\left(1+\xi_{1}^{6}+\xi_{2}^{2}+\varphi\left(x_{2}\right) \xi_{2}^{6}\right)^{2}} .
$$

In this case, $\|\alpha\|=0$ and $\left(1+\rho_{A^{*}}\left(\xi_{1}, \xi_{2}\right)\right)^{-\|\alpha\|}=1$. Since $\left|\varphi^{\prime}\left(x_{2}\right)\right| \leq C_{3}$ and $\varphi\left(x_{2}\right)>C_{1}$, we immediately see that

$$
\frac{\left|\varphi^{\prime}\left(x_{2}\right)\right| \xi_{2}^{6}\left(1+\xi_{2}^{2}\right)}{\left(1+\xi_{1}^{6}+\xi_{2}^{2}+\varphi\left(x_{2}\right) \xi_{2}^{6}\right)^{2}} \leq C_{3} \frac{\xi_{2}^{6}}{1+\varphi\left(x_{2}\right) \xi_{2}^{6}} \leq \frac{C_{3}}{C_{1}}
$$

3. $(\beta, \alpha)=(0,0,1,0)$. We have

$$
\left|\partial_{\xi_{1}} \sigma(x, \xi)\right| \lesssim \frac{\left|\xi_{1}\right|^{5}\left(1+\xi_{2}^{2}\right)}{\left(1+\xi_{1}^{6}+\xi_{2}^{2}+\varphi\left(x_{2}\right) \xi_{2}^{6}\right)^{2}}
$$

We expect our estimates to be $\lesssim\left(1+\left|\xi_{1}\right|^{2}\right)^{-1 / 2}$ or $\lesssim\left(1+\left|\xi_{2}\right|^{2 / 3}\right)^{-1 / 2}$. Indeed, if $\left|\xi_{1}\right|^{3} \geq\left|\xi_{2}\right|$, then

$$
\frac{\left|\xi_{1}\right|^{5}\left(1+\xi_{2}^{2}\right)}{\left(1+\xi_{1}^{6}+\xi_{2}^{2}+\varphi\left(x_{2}\right) \xi_{2}^{6}\right)^{2}} \lesssim \frac{\left|\xi_{1}\right|^{5}}{1+\xi_{1}^{6}} \lesssim\left(1+\left|\xi_{1}\right|\right)^{-1} \lesssim\left(1+\left|\xi_{1}\right|^{2}\right)^{-1 / 2},
$$

while if $\left|\xi_{1}\right|^{3} \leq\left|\xi_{2}\right|$, we have

$$
\frac{\left|\xi_{1}\right|^{5}\left(1+\xi_{2}^{2}\right)}{\left(1+\xi_{1}^{6}+\xi_{2}^{2}+\varphi\left(x_{2}\right) \xi_{2}^{6}\right)^{2}} \lesssim \frac{\left|\xi_{2}\right|^{5 / 3}}{1+\xi_{2}^{2}} \lesssim\left(1+\left|\xi_{2}\right|\right)^{-1 / 3} \lesssim\left(1+\left|\xi_{2}\right|^{2 / 3}\right)^{-1 / 2} .
$$

4. $(\beta, \alpha)=(0,0,0,1)$. We have

$$
\left|\partial_{\xi_{2}} \sigma(x, \xi)\right| \lesssim \frac{\left|\xi_{2}\right|\left(\xi_{1}^{6}+\xi_{2}^{6}+\xi_{2}^{4}\right)}{\left(1+\xi_{1}^{6}+\xi_{2}^{2}+\varphi\left(x_{2}\right) \xi_{2}^{6}\right)^{2}}
$$


Note that $\xi_{2}^{4}=\sqrt{\xi_{2}^{2} \xi_{2}^{6}} \lesssim \xi_{2}^{2}+\varphi\left(x_{2}\right) \xi_{2}^{6}$. Thus,

$$
\frac{\xi_{1}^{6}+\xi_{2}^{6}+\xi_{2}^{4}}{1+\xi_{1}^{6}+\xi_{2}^{2}+\varphi\left(x_{2}\right) \xi_{2}^{6}} \lesssim 1
$$

Now, if $\left|\xi_{1}\right|^{3} \geq\left|\xi_{2}\right|$, then

$$
\frac{\left|\xi_{2}\right|\left(\xi_{1}^{6}+\xi_{2}^{6}+\xi_{2}^{4}\right)}{\left(1+\xi_{1}^{6}+\xi_{2}^{2}+\varphi\left(x_{2}\right) \xi_{2}^{6}\right)^{2}} \lesssim \frac{\left|\xi_{1}\right|^{3}}{1+\xi_{1}^{6}} \lesssim\left(1+\left|\xi_{1}\right|^{3}\right)^{-1} \lesssim\left(1+\left|\xi_{1}\right|^{2}\right)^{-3 / 2}
$$

Finally, if $\left|\xi_{1}\right|^{3} \leq\left|\xi_{2}\right|$, we have

$$
\frac{\left|\xi_{2}\right|\left(\xi_{1}^{6}+\xi_{2}^{6}+\xi_{2}^{4}\right)}{\left(1+\xi_{1}^{6}+\xi_{2}^{2}+\varphi\left(x_{2}\right) \xi_{2}^{6}\right)^{2}} \lesssim \frac{\left|\xi_{2}\right|}{1+\xi_{2}^{2}} \lesssim\left(1+\left|\xi_{2}\right|\right)^{-1} \lesssim\left(1+\left|\xi_{2}\right|^{2 / 3}\right)^{-3 / 2} .
$$

We divide the proof of Theorem 3.1 into several subsections in which we explain our strategy leading to the conclusion we wish to achieve. We will start by showing that we can reduce the $L^{2}$ boundedness of $\sigma(x, D)$ to the $L^{2}$ boundedness of pseudodifferential operators with so called anisotropic elementary symbols. Finally, we will prove that any elementary symbol yields an $L^{2}$ bounded pseudodifferential operator.

We will repeatedly use the following elementary lemma, see $[2,3,4]$.

Lemma 3.2. Suppose $A$ is an expansive matrix, and $\lambda_{-}$and $\lambda_{+}$are any positive real numbers such that $1<\lambda_{-}<\min _{\lambda \in \sigma(A)}|\lambda|$ and $\max _{\lambda \in \sigma(A)}|\lambda|<\lambda_{+}<b=|\operatorname{det} A|$. Then, there exists $c>0$ such that

$$
\begin{array}{ll}
(1 / c)\left(\lambda_{-}\right)^{j}|x| \leq\left|A^{j} x\right| \leq c\left(\lambda_{+}\right)^{j}|x| & \text { for } j \geq 0, \\
(1 / c)\left(\lambda_{+}\right)^{j}|x| \leq\left|A^{j} x\right| \leq c\left(\lambda_{-}\right)^{j}|x| & \text { for } j \leq 0 .
\end{array}
$$

Furthermore, if $A$ is diagonalizable over $\mathbb{C}$, then we may take $\lambda_{-}=\min _{\lambda \in \sigma(A)}|\lambda|$ and $\lambda_{+}=\max _{\lambda \in \sigma(A)}|\lambda|$.

3.1. Elementary symbols. We want to reduce our study to that of anisotropic elementary symbols. Assume that such a reduction is possible. Then, we will only need to worry (in a subsequent subsection) about the $L^{2}$ boundedness of $\sigma(x, D)$ with anisotropic elementary symbol of the form

$$
\sigma(x, \xi)=\sum_{j=0}^{\infty} m_{j}(x) \widehat{\phi}\left(\left(A^{*}\right)^{-j} \xi\right)
$$

where $m_{j}$ are bounded and satisfying appropriate smoothness and decay, and $\widehat{\phi} \in \mathcal{S}$ is supported away from the origin, that is, there exists $R>0$ such that

$$
\operatorname{supp} \widehat{\phi} \subset\left\{\xi \in \mathbb{R}^{n}: 1 / R<|\xi|<R\right\} .
$$

We begin by stating the following claim.

Claim 1. There exists (a fixed) $J \geq 1$ such that

$$
\operatorname{supp} \widehat{\phi}\left(\left(A^{*}\right)^{-j} \cdot\right) \cap \operatorname{supp} \widehat{\phi}\left(\left(A^{*}\right)^{-k} \cdot\right)=\emptyset,
$$

for $|j-k| \geq J$. 
This non-overlapping property of the supports will play an important role in our arguments. The proof of the claim is a consequence of the elementary Lemma 3.2. Letting $\phi_{j}(x)=|\operatorname{det} A|^{j} \phi\left(A^{j} x\right)$, we have $\widehat{\phi}_{j}(\xi)=\widehat{\phi}\left(\left(A^{*}\right)^{-j} \xi\right)$. Hence, if $j \geq 0$, Lemma 3.2 implies that

$$
\begin{aligned}
\operatorname{supp} \widehat{\phi}_{j} & \subset\left\{\xi \in \mathbb{R}^{n}: 1 / R<\left|\left(A^{*}\right)^{-j} \xi\right|<R\right\} \\
& \subset\left\{\xi \in \mathbb{R}^{n}:\left(\lambda_{-}\right)^{j} /(R c)<|\xi|<R c\left(\lambda_{+}\right)^{j}\right\} .
\end{aligned}
$$

Let $J$ be the smallest positive integer such that $\left(\lambda_{-}\right)^{J}>R^{2} c$. By the previous inclusion of supports,

$$
\operatorname{supp} \widehat{\phi} \cap \operatorname{supp} \widehat{\phi}_{j}=\emptyset \quad \text { for } j>J .
$$

Thus, applying supp $\widehat{\phi}_{k}=\left(A^{*}\right)^{k}(\operatorname{supp} \widehat{\phi})$ yields

$$
\operatorname{supp} \widehat{\phi}_{k} \cap \operatorname{supp} \widehat{\phi}_{j}=\emptyset \quad \text { for }|j-k|>J,
$$

and the claim is proved.

Returning now to our elementary symbols, if we let $f_{j}=f * \phi_{j}$, for some $f \in \mathcal{S}$, then $\widehat{f}_{j}=\widehat{f}_{j}$. Using Plancherel's theorem (twice) and (3.6) (that guarantees the sums are finite), we get

$$
\sum_{j \geq 0}\left\|f_{j}\right\|_{L^{2}}^{2}=\sum_{j \geq 0}\left\|\widehat{f}_{j}\right\|_{L^{2}}^{2} \lesssim\|\widehat{\phi}\|_{L^{\infty}}^{2}\|f\|_{L^{2}}^{2}
$$

Furthermore,

$$
\sigma(x, D) f(x)=\sum_{j \geq 0} m_{j}(x) f_{j}(x) .
$$

Therefore, the proof of the $L^{2}$ boundedness of $\sigma(x, D)$ with elementary symbol (3.5) reduces to showing the following inequality:

$$
\left\|\sum_{j \geq 0} m_{j} f_{j}\right\|_{L^{2}} \leq C\left(\sum_{j \geq 0}\left\|f_{j}\right\|_{L^{2}}^{2}\right)^{1 / 2} .
$$

We will come back to (3.7) in Subsection 3.3. For now, we simply note that the constant $C>0$ is determined by the reduction of a generic symbol to an elementary one, and, in particular, from the control on $m_{j}$, control that will be obtained from the symbol belonging to $S_{1,0}^{0}(A)$.

3.2. Reduction to elementary symbols. We indicate how the reduction to elementary symbols is performed. For a given $u \in \mathcal{S}$, such that $v=\widehat{u}$ is compactly supported away from the origin,

$$
\operatorname{supp} v \subset\{\xi: 1 / R<|\xi|<R\},
$$

we write $u_{j}(x)=|\operatorname{det} A|^{j} u\left(A^{j} x\right)$, and we have

$$
\widehat{u}_{j}(\xi)=\widehat{u}\left(\left(A^{*}\right)^{-j} \xi\right)=v\left(\left(A^{*}\right)^{-j} \xi\right):=v^{j}(\xi) .
$$

Assume that

$$
\sum_{j \in \mathbb{Z}} v^{j}(\xi)=1, \xi \neq 0
$$


Given an arbitrary symbol $\sigma \in S_{1,0}^{0}(A)$, if we further assume that $\sigma(x, 0)=0$, then we can write

$$
\sigma=\sum_{j=0}^{\infty} \sigma_{j}+\tau
$$

where

$$
\sigma_{j}(x, \xi)=v^{j}(\xi) \sigma(x, \xi), j \geq 0
$$

and

$$
\tau(x, \xi)=\sum_{j=-\infty}^{-1} v^{j}(\xi) \sigma(x, \xi) .
$$

Note that the assumption $\sigma(x, 0)=0$ does not imply a further restriction on the $L^{p}$ boundedness properties of $\sigma(x, D)$. Indeed, if $\sigma(x, 0) \neq 0$, then we let

$$
\tilde{\sigma}(x, \xi)=\sigma(x, \xi)-\sigma(x, 0) .
$$

Now, we simply notice that $\tilde{\sigma}(x, 0)=0$ and $\sigma(x, 0)$ is a smooth and bounded multiplier; indeed, the pseudodifferential operator $T$ associated to $\sigma(x, 0)$ is given by $T f=\sigma(\cdot, 0) \cdot f$, and, by condition (3.1) with $k=0$, we can bound $\|T f\|_{L^{p}} \leq$ $\|\sigma(\cdot, 0)\|_{L_{x}^{\infty}}\|f\|_{L^{p}}$. Therefore, the $L^{p}$ boundedness properties of $\sigma(x, D)$ and $\tilde{\sigma}(x, D)$ are equivalent.

3.2.1. The "negative" part of the multiplier. We wish to show next that the multiplier operator $\tau(x, D)$ is bounded on Lebesgue spaces. We start by noting that $\tau(x, \xi)$ vanishes on $|\xi| \geq c R$, where $R>0$ is determined by the support of $v$ and $c>0$ is the constant in Lemma 3.2. Indeed, for $j \leq-1$ and $|\xi| \geq c R$,

$$
\left|\left(A^{*}\right)^{-j} \xi\right| \geq \frac{1}{c}\left(\lambda_{-}\right)^{-j}|\xi|>R
$$

that is $\xi \notin \operatorname{supp} v^{j}$. Recall also that the functions $v^{j}$ have non-overlapping supports (see Claim 1 in Subsection 3.1):

$$
\operatorname{supp} v^{j} \cap \operatorname{supp} v^{k}=\emptyset,|j-k|>J,
$$

where $J$ is the smallest positive integer such that $\left(\lambda_{-}\right)^{J}>c R^{2}$. Therefore, we can simply concentrate on the properties of $\tau(x, \xi)$ at the frequency scale $1 / R \leq\left|\left(A^{*}\right)^{-j} \xi\right| \leq$ $R$, for a fixed $j \leq-1$. With the exception of (possibly) a finite number of terms at a proportional scale $k$ determined by $|k-j| \leq J$, all the other terms in the expression of $\tau$ will vanish. by

The pseudodifferential operator $\tau(x, D)$ can also be represented in its kernel form

$$
\tau(x, D) f(x)=\int K(x, y) f(y) d y
$$

where

$$
K(x, y)=\int \tau(x, \xi) e^{i \xi \cdot(x-y)} d \xi
$$


The integration in $\xi$ is over the compact set $\{\xi:|\xi| \leq c R\}$. Using integration by parts, we can write

$$
K(x, y)=\left(1+|x-y|^{2}\right)^{-M} L_{M}(x, y),
$$

where $L_{M}$ is a bounded smooth function and $M$ is arbitrarily large. From here, we immediately get that $\tau(x, D)$ is bounded on $L^{p}$. We briefly indicate why the statement about $L_{M}$ is true.

It is sufficient to prove that

$$
\left\|\partial_{\xi}^{\beta} \tau(x, \xi)\right\|_{L^{\infty}} \lesssim 1
$$

for $|\xi| \leq c R$, which is equivalent to prove that, for $j \leq-1$ and $1 / R \leq\left|\left(A^{*}\right)^{-j} \xi\right| \leq R$,

$$
\left\|\partial_{\xi}^{\beta}\left[v^{j}(\xi) \sigma(x, \xi)\right]\right\|_{L^{\infty}} \lesssim c_{j}
$$

We show why this is true only for the first order derivative. Note that

$$
v^{j}(\xi) \sigma(x, \xi)=v^{j}(\xi) \sigma_{x}(\xi)=w_{x}\left(\left(A^{*}\right)^{-j} \xi\right),
$$

where

$$
w_{x}(\cdot)=v(\cdot) \sigma_{x}\left(\left(A^{*}\right)^{j} \cdot\right) .
$$

Since $\sigma \in S_{1,0}^{0}(A)$, from the inequalities (3.1), we have

$$
\left|\partial_{\xi}^{\beta} \sigma_{x}\left[\left(A^{*}\right)^{j} \cdot\right]\left(\left(A^{*}\right)^{-j} \xi\right)\right| \leq C_{\beta} .
$$

Therefore,

$$
\begin{aligned}
\left|\partial_{\xi}\left[v^{j}(\xi) \sigma_{x}(\xi)\right]\right| & =\left|\partial_{\xi}\left[w_{x}\left(\left(A^{*}\right)^{-j} \xi\right)\right]\right| \\
& \leq\left\|\left(A^{*}\right)^{-j}\right\|\left(\left\|\partial_{\xi} v\right\|_{L^{\infty}}\left|\sigma_{x}(\xi)\right|+\|v\|_{L^{\infty}}\left|\partial_{\xi} \sigma_{x}\left[\left(A^{*}\right)^{j} \cdot\right]\left(\left(A^{*}\right)^{-j} \xi\right)\right|\right) \\
& \lesssim\left\|\left(A^{*}\right)^{-j}\right\| \lesssim 1 .
\end{aligned}
$$

3.2.2. The "positive" part of the multiplier. The previous discussion indicates that we can safely concentrate on the "positive" part of the given symbol, namely $\lambda(x, \xi)=$ $\sum_{j \geq 0} \sigma_{j}(x, \xi)$. We will show that, through a periodization argument, we can decompose the positive part of $\sigma$ into a convergent sum of elementary symbols.

For $j \geq 0$, let

$$
\Lambda_{j}(x, \xi)=\sum_{k \in \mathbb{Z}^{n}} \sigma_{j}\left(x,\left(A^{*}\right)^{j}(\xi-2 \pi R k)\right),
$$

where $R$ is the positive real that determines the support of $v$ (see previous subsection). Recall that

$$
\sigma_{j}(x, \xi)=v^{j}(\xi) \sigma(x, \xi)=v\left(\left(A^{*}\right)^{-j} \xi\right) \sigma(x, \xi) .
$$

Due to the support condition on $v$, it is easy to see that $\sigma_{j}\left(\left(x, A^{*}\right)^{j} \xi\right)$ vanishes on $|\xi| \geq R$. Note also that $\Lambda_{j}$ is a $2 \pi R \mathbb{Z}^{n}$ periodic function (as a function of $\xi$, for a fixed $x$ ).

Let now $\psi \in C^{\infty}$ be compactly supported,

$$
\operatorname{supp} \psi \subset\left\{\xi: R^{-1}-\epsilon<|\xi|<R+\epsilon\right\},
$$

where $0<\epsilon<1 /(12 R)$, and such that $\psi v=v$ on $\operatorname{supp} v$. Then clearly,

$$
\sigma_{j}(x, \xi)=\psi\left(\left(A^{*}\right)^{-j} \xi\right) \sigma_{j}(x, \xi)=\psi^{j}(\xi) \sigma_{j}(x, \xi) .
$$


Furthermore, we have

$$
\sigma_{j}(x, \xi)=\psi^{j}(\xi) \Lambda_{j}\left(x,\left(A^{*}\right)^{-j} \xi\right)
$$

Indeed, we can write

$$
\Lambda_{j}\left(x,\left(A^{*}\right)^{-j} \xi\right)=\sigma_{j}(x, \xi)+\sum_{|k| \neq 0} \sigma_{j}\left(x, \xi-2 \pi R\left(A^{*}\right)^{j} k\right),
$$

and we distinguish two cases.

Case 1: $\left|\left(A^{*}\right)^{-j} \xi\right|>R+\epsilon$

Then $v^{j}(\xi)=v\left(\left(A^{*}\right)^{-j} \xi\right)=0$, therefore $\sigma_{j}(x, \xi)=0$, and $\psi^{j}(\xi)=0$. Consequently, (3.12) holds.

Case 2: $\left|\left(A^{*}\right)^{-j} \xi\right| \leq R+\epsilon$

Then, for $|k| \neq 0$, we have

$$
\mid\left(A^{*}\right)^{-j}\left(\xi-2 \pi R\left(\left(A^{*}\right)^{j} k\right)|\geq 2 \pi R| k|-|\left(A^{*}\right)^{-j} \xi|\geq 2 \pi R| k \mid-R-\epsilon>R .\right.
$$

This, in turn, implies that

$$
\sigma_{j}\left(x, \xi-2 \pi R\left(\left(A^{*}\right)^{j} k\right)\right)=0,
$$

and again we have the equality (3.12).

If we now expand $\Lambda_{j}$ into its Fourier series, we have

$$
\Lambda_{j}(x, \xi)=\sum_{k \in \mathbb{Z}^{n}} c_{j k}(x) e^{-i k \cdot \xi / R},
$$

where

$$
c_{j k}(x)=(2 \pi R)^{-n} \int_{[-\pi R, \pi R]^{n}} \sigma_{j}\left(x,\left(A^{*}\right)^{j} \xi\right) e^{-i k \cdot \xi / R} d \xi .
$$

Using (3.12), we can further write

$$
\sigma_{j}(x, \xi)=\sum_{k \in \mathbb{Z}^{n}}\left(1+|k|^{2}\right)^{-(n+1) / 2} \kappa_{j k}(x) e^{-i k \cdot\left(A^{*}\right)^{-j} \xi / R} \psi\left(\left(A^{*}\right)^{-j} \xi\right),
$$

where

$$
\kappa_{j k}(x)=\left(1+|k|^{2}\right)^{(n+1) / 2} c_{j k}(x) .
$$

Therefore, we see that

$$
\lambda(x, \xi)=\sum_{j \geq 0} \sigma_{j}(x, \xi)=\sum_{k \in \mathbb{Z}^{n}}\left(1+|k|^{2}\right)^{-(n+1) / 2} \sigma^{k}(x, \xi),
$$

where

$$
\left.\sigma^{k}(x, \xi)=\sum_{j=0}^{\infty} \kappa_{j k}(x) e^{-i k \cdot\left(A^{*}\right)^{-j} \xi / R} \psi\left(\left(A^{*}\right)^{-j} \xi\right)=\sum_{j=0}^{\infty} \kappa_{j k}(x) \widehat{\phi}^{k}\left(A^{*}\right)^{-j} \xi\right) .
$$

It is clear then that it is sufficient to prove the uniform boundedness (with respect to the index $k$ ) of the multiplier operators $T_{\sigma^{k}}$, since

$$
\|T\|_{L^{2} \rightarrow L^{2}} \leq \sum_{k \in \mathbb{Z}^{n}}\left(1+|k|^{2}\right)^{-(n+1) / 2}\left\|T_{\sigma^{k}}\right\|_{L^{2} \rightarrow L^{2}} .
$$


The multipliers $\sigma^{k}$ are our typical elementary symbols (compare to (3.5)). The only thing left is to obtain the correct control on the coefficients $\kappa_{j k}$ that would allow us to conclude with an inequality like (3.7). This control is determined by the estimates on the symbol $\sigma \in S_{1,0}^{0}(A)$. Recall that

$$
c_{j k}(x)=(2 \pi R)^{-n} \int e^{-i k \cdot \xi / R} \sigma_{j}\left(x,\left(A^{*}\right)^{j} \xi\right) d \xi .
$$

If we integrate by parts, we can gain $|k|^{-N}$ (for $N$ as large as we please) as long as we can bound $\partial_{\xi}^{N} \sigma_{j}\left(x,\left(A^{*}\right)^{j} \xi\right)$. This in turn, will imply that the coefficients $\kappa_{j k}$ are bounded (again, compare with (3.5) where we required that the $m_{j}$ coefficients are bounded). We prove that we have indeed the right control on the coefficients as follows.

Lemma 3.3. Suppose that the symbol $\sigma$ is in the class $S_{1,0}^{0}(A)$. Let $v$ be a $C^{\infty}$ function such that $\operatorname{supp} v \subset\{\xi: 1 / R<|\xi|<R\}$. For each $j \in \mathbb{Z}$, define

$$
\sigma^{j}(x, \xi)=\sigma\left(x,\left(A^{*}\right)^{j} \xi\right) .
$$

Then, for all $\beta$ and some constants $C_{\beta}>0$,

$$
\left\|\partial^{\beta}\left(v \sigma^{j}\right)\right\|_{L_{\xi}^{\infty}} \leq C_{\beta} .
$$

Proof. By the condition on the support of $v$ and by the product rule we have

$$
\left\|\partial_{\xi}^{\beta}\left(v \sigma^{j}\right)\right\|_{L_{\xi}^{\infty}}=\sup _{1 / R<|\xi|<R}\left|\partial^{\beta}\left(v \sigma_{x}^{j}\right)(\xi)\right| \leq C \sup _{|\alpha| \leq|\beta|} \sup _{1 / R<|\xi|<R}\left|\partial^{\alpha} \sigma_{x}^{j}(\xi)\right| .
$$

Given any $\xi$ in the annulus $1 / R<|\xi|<R$, define $\eta=\left(A^{*}\right)^{j} \xi$. Note that $\rho_{A^{*}}(\eta) \sim$ $|\operatorname{det} A|^{j}$. Hence, by (3.1)

$$
\left|\partial^{\alpha} \sigma_{x}^{j}\left(\left(A^{*}\right)^{-j} \eta\right)\right|=\left|\partial^{\alpha} \sigma_{x}^{j}(\xi)\right| \leq C_{\alpha} .
$$

This completes the proof of the lemma.

Corollary 3.4. For any $N>0$ and for all $x$, we have

$$
c_{j k}(x)=(2 \pi R)^{-n} \int e^{-i k \cdot \xi / R} v(\xi) \sigma^{j}(x, \xi) d \xi=O\left(|k|^{-N}\right) \text { as }|k| \rightarrow \infty
$$

and $c_{j k}(x)=O(1)$ as $|j| \rightarrow \infty$.

Remark 3.1. The previous corollary translates into saying that the coefficients of an elementary symbols, which we denoted by $m_{j}$, are smooth and bounded. In fact, a completely analogous argument to the one employed in Lemma 3.3 shows that we can control the $L^{\infty}$ norm of the derivatives of the coefficients and get that

$$
\left\|\partial^{\alpha} m_{j}\right\|_{L^{\infty}} \leq C_{\alpha}
$$

for all multi-indices $\alpha$ and some constants $C_{\alpha}>0$. 
3.3. Boundedness of elementary pseudodifferential operators. We come back now to inequality (3.7). We wish to show that for a family $\left(m_{j}\right)$ of appropriately smooth and bounded functions, and $\left(f_{j}\right) \in L^{2}$ such that supp $\widehat{f}_{k} \cap \operatorname{supp} \widehat{f}_{j}=\emptyset$ for $\mid j-$ $k \mid>J$, we have

$$
\left\|\sum_{j \geq 0} m_{j} f_{j}\right\|_{L^{2}} \leq C\left(\sum_{j \geq 0}\left\|f_{j}\right\|_{L^{2}}^{2}\right)^{1 / 2} .
$$

Our argument is based on the following decomposition of the coefficients $m_{j}, j \geq 0$ into their "good" and "bad" parts; our decompositions is a slight modification of the one that appears in [12].

Claim 2. We can write $m_{j}=g_{j}+b_{j}$, where $\left\|g_{j}\right\|_{L^{\infty}} \lesssim 1,\left\|b_{j}\right\|_{L^{\infty}} \lesssim\left(\lambda_{-}\right)^{-j}$, and $\operatorname{supp} \widehat{f_{j} g_{j}} \subset\left\{\xi:(2 R)^{-1}<\left|\left(A^{*}\right)^{-j} \xi\right|<R+(2 R)^{-1}\right\}$.

The proof of the claim is as follows. Consider $K \in C_{c}^{\infty}$ such that $\widehat{K}(0)=1$ and $\widehat{K}(\xi)=0$ on $|\xi|>(2 R)^{-1}$. Define as usual

$$
K_{j}(x)=|\operatorname{det} A|^{j} K\left(A^{j} x\right)=b^{j} K\left(A^{j} x\right),
$$

and let

$$
g_{j}=K_{j} * m_{j}, \text { and } b_{j}=m_{j}-g_{j}
$$

By construction, $m_{j}=g_{j}+b_{j}$. The estimate of the good part is easy, since clearly

$$
\left\|g_{j}\right\|_{L^{\infty}} \leq\|K\|_{L^{1}}\left\|m_{j}\right\|_{L^{\infty}}
$$

To control the bad part, we use the Mean Value Theorem and the fact that $\int K_{j}(u)=$ $\int K(u)=\widehat{K}(0)=1$ to get

$$
\begin{aligned}
\left|b_{j}(x)\right| & \leq \int\left|m_{j}(x)-m_{j}(x-u)\right|\left|K_{j}(u)\right| d u \lesssim \int|u| b^{j}\left|K\left(A^{j} u\right)\right| d u \\
& \lesssim \int\left|A^{-j} u\right||K(u)| d u \leq c\left(\lambda_{-}\right)^{-j} \int|u||K(u)| d u \lesssim\left(\lambda_{-}\right)^{-j} .
\end{aligned}
$$

Finally, we show the inclusion of supports. Let us assume first that $m_{j} \in L^{2}$. Then $g_{j} \in L^{2}$ and

Since

$$
\operatorname{supp} \widehat{f_{j} g_{j}} \subseteq \operatorname{supp} \widehat{f}_{j}+\operatorname{supp} \widehat{g}_{j} \subseteq \operatorname{supp} \widehat{f}_{j}+\operatorname{supp} \widehat{K}_{j}
$$

$$
\operatorname{supp} \widehat{f_{j}} \subset\left\{\xi: R^{-1}<\left|\left(A^{*}\right)^{-j} \xi\right|<R\right\} \text { and supp } \widehat{K}_{j} \subset\left\{\xi:\left|\left(A^{*}\right)^{-j} \xi\right|<(2 R)^{-1}\right\},
$$

we conclude that

$$
\operatorname{supp} \widehat{f_{j} g_{j}} \subset\left\{\xi:(2 R)^{-1}<\left|\left(A^{*}\right)^{-j} \xi\right|<R+(2 R)^{-1}\right\} .
$$

If $m_{j} \notin L^{2}$, we approximate it with the cutoffs $m_{j} \chi_{\{|\xi|<N\}}$ and then let $N \rightarrow \infty$ to arrive to the same conclusion.

In order to prove (3.13), it therefore suffices to estimate

$$
\left\|\sum_{j \geq 0} b_{j} f_{j}\right\|_{L^{2}} \quad \text { and } \quad\left\|\sum_{j \geq 0} g_{j} f_{j}\right\|_{L^{2}} .
$$


We utilize the estimates shown in Claim 2 and the Cauchy-Schwarz inequality.

$$
\begin{aligned}
& \left\|\sum_{j \geq 0} b_{j} f_{j}\right\|_{L^{2}} \leq \sum_{j \geq 0}\left\|b_{j}\right\|_{L^{\infty}}\left\|f_{j}\right\|_{L^{2}} \leq\left(\sum_{j \geq 0}\left\|b_{j}\right\|_{L^{\infty}}^{2}\right)^{1 / 2}\left(\sum_{j \geq 0}\left\|f_{j}\right\|_{L^{2}}^{2}\right)^{1 / 2} \\
& \lesssim\left(\sum_{j \geq 0}\left(\lambda_{-}\right)^{-2 j}\right)^{1 / 2}\left(\sum_{j \geq 0}\left\|f_{j}\right\|_{L^{2}}^{2}\right)^{1 / 2}=\lambda_{-}\left(\left(\lambda_{-}\right)^{2}-1\right)^{-1 / 2}\left(\sum_{j \geq 0}\left\|f_{j}\right\|_{L^{2}}^{2}\right)^{1 / 2} .
\end{aligned}
$$

With the notation in Lemma 3.2, let $I$ be the smallest positive integer such that $\left(\lambda_{-}\right)^{I} \geq c\left(2 R^{2}+1\right)$. Then, for all $i>I$, we have

$$
\left\{\xi:(2 R)^{-1}<|\xi|<R+(2 R)^{-1}\right\} \cap\left\{\xi:(2 R)^{-1}<\left|\left(A^{*}\right)^{-i} \xi\right|<R+(2 R)^{-1}\right\}=\emptyset .
$$

By applying $A^{* k}$, we conclude that

$$
\operatorname{supp} \widehat{f_{k} g_{k}} \cap \operatorname{supp} \widehat{f_{i} g_{i}}=\emptyset \text {, for all }|i-k|>I \text {. }
$$

Therefore,

$$
\begin{aligned}
\left\|\sum_{j \geq 0} g_{j} f_{j}\right\|_{L^{2}} & \leq \sum_{i=0}^{I-1}\left\|\sum_{k \geq 0} g_{i+I k} f_{i+I k}\right\|_{L^{2}} \leq I\left(\sum_{j \geq 0}\left\|g_{j} f_{j}\right\|_{L^{2}}^{2}\right)^{1 / 2} \\
& \lesssim I\|K\|_{L^{1}}\left(\sum_{j \geq 0}\left\|f_{j}\right\|_{L^{2}}^{2}\right)^{1 / 2} .
\end{aligned}
$$

Estimates (3.16) and (3.17) yield estimate (3.13), thus finishing the proof of Theorem 3.1.

It is worth noting that the same proof works with minor modifications if we assume that $\sigma \in S_{1, \delta}^{0}(A), 0 \leq \delta<1$. We end the paper by exhibiting an example of an anisotropic symbol in $S_{1,1}^{0}(A)$ which is unbounded on $L^{2}$.

Example 3.2. Let $0 \neq v \in \mathbb{R}^{n}$ be fixed. Let $\varphi$ be a smooth bump such that

$$
\operatorname{supp} \varphi \subset B(v, \delta) \text {, and } \varphi(\xi)=1 \text { on } B(v, \delta / 2)
$$

for some $\delta>0$, where $B(v, \delta)=\left\{\xi \in \mathbb{R}^{n}:|\xi-v|<\delta\right\}$. Since the matrix $A^{*}$ is expansive we can choose $\delta>0$ sufficiently small such that the dilated balls $\left(A^{*}\right)^{j}(B(v, \delta))$ are disjoint for $j \in \mathbb{Z}$. Define the symbol

$$
\sigma(x, \xi)=\sum_{j=1}^{\infty} e^{-i x \cdot\left(A^{*}\right)^{j} v} \varphi\left(\left(A^{*}\right)^{-j} \xi\right) .
$$

Due to our choice of $\delta>0$, we notice that for every $\xi \in \mathbb{R}^{n}$, this summation contains at most one non-zero term. Recall that $\sigma \in S_{1,1}^{0}(A)$ if

$$
\left|\partial_{x}^{\alpha} \partial_{\xi}^{\beta}\left[\sigma\left(A^{-k} \cdot, A^{* k} \cdot\right)\right]\left(A^{k} x,\left(A^{*}\right)^{-k} \xi\right)\right| \leq C_{\alpha, \beta},
$$

for all $(x, \xi) \in \mathbb{R}^{n} \times \mathbb{R}^{n} \backslash\{0\}$ and $k \in \mathbb{Z}$ is such that $1+\rho_{A^{*}}(\xi) \sim b^{k}$. Observe that $\varphi\left(\left(A^{*}\right)^{-j} \xi\right) \neq 0$ implies that $\xi \in\left(A^{*}\right)^{j}(B(v, \delta))$ and thus $\rho_{A^{*}}(\xi) \sim b^{j}$. Thus, the dilates in (3.19) undo those present in (3.18), which shows that $\sigma \in S_{1,1}^{0}(A)$. 
Next, we shall show that for the symbol considered, $\sigma(x, D): L^{2} \not \rightarrow L^{2}$. Fix $f \in \mathcal{S}$ such that supp $\widehat{f} \subset B(0, r)$ for some $r>0$. For $N \in \mathbb{N}$, define

$$
F_{N}(x)=\sum_{j=1}^{N} \frac{1}{j} e^{i x \cdot\left(A^{*}\right)^{j} v} f(x) .
$$

Letting $f_{j}(x)=e^{i x \cdot\left(A^{*}\right)^{j} v} f(x)$, we see that $\widehat{f}_{j}(\xi)=\widehat{f}\left(\xi-\left(A^{*}\right)^{j} v\right)$, and $\operatorname{supp} \widehat{f}_{j} \subset$ $B\left(\left(A^{*}\right)^{j} v, r\right)$. Since $A^{*}$ is expansive we can choose $r>0$ sufficiently small such that $B\left(\left(A^{*}\right)^{j} v, r\right) \subset\left(A^{*}\right)^{j}(B(v, \delta / 2))$ for all $j \in \mathbb{N}$. This automatically yields that

$$
\operatorname{supp} \hat{f}_{j} \cap \operatorname{supp} \hat{f}_{k}=\emptyset \quad \text { for } j \neq k,
$$

and that for all $j \in \mathbb{N}$,

$$
\xi \in \operatorname{supp} \widehat{f}_{j} \Longrightarrow \varphi_{j}(\xi)=1 .
$$

Hence, by the orthogonality of $f_{j}$ 's

$$
\left\|F_{N}\right\|_{L^{2}}=\left(\sum_{j=1}^{N} \frac{1}{j^{2}}\left\|f_{j}\right\|_{L^{2}}^{2}\right)^{1 / 2} \leq \frac{\pi}{\sqrt{6}}\|f\|_{L^{2}} .
$$

Finally, since $\sigma(x, \xi)=e^{-i x \cdot\left(A^{*}\right)^{j} v}$ for $\xi \in \operatorname{supp} \varphi_{j}$, by the Fourier inversion formula we have

$$
\begin{aligned}
\sigma(x, D)\left(F_{N}\right)(x) & =\int_{\mathbb{R}^{n}} \sigma(x, \xi) e^{i x \cdot \xi} \sum_{j=1}^{N} \frac{1}{j} \widehat{f}\left(\xi-\left(A^{*}\right)^{j} v\right) d \xi \\
& =\sum_{j=1}^{N} \frac{1}{j} \int_{\mathbb{R}^{n}} e^{i x \cdot\left(\xi-\left(A^{*}\right)^{j} v\right)} \widehat{f}\left(\xi-\left(A^{*}\right)^{j} v\right) d \xi=\frac{1}{(2 \pi)^{n}}\left(\sum_{j=1}^{N} \frac{1}{j}\right) f .
\end{aligned}
$$

Thus, there exists constant $C>0$, such that for any $N \in \mathbb{N}$,

$$
\left\|\sigma(x, D) F_{N}\right\|_{L^{2}} \geq C \log N\|f\|_{L^{2}} \geq \frac{C \sqrt{6}}{\pi}\left\|F_{N}\right\|_{L^{2}}
$$

This proves that $\sigma(x, D)$ is not bounded on $L^{2}$.

Remark 3.2. We have demonstrated that the anisotropic class of inhomogeneous symbols $S_{\delta, \delta}^{0}(A), 0 \leq \delta<1$, shares similar $L^{2}$ boundedness results with its isotropic counterpart. This comment also applies to the $L^{p}$ boundedness, $1<p<\infty$, of the smaller anisotropic class $S_{1, \delta}^{0}(A), 0 \leq \delta<1$. The next natural step would be a systematic study of the $L^{p}$ boundedness properties of more exotic anisotropic classes of symbols $S_{\gamma, \delta}^{m}(A)$, as carried out in the isotropic setting by Fefferman [8] or Miyachi [15]. However, this goes beyond the scope of this paper which merely aimed at showing plausibility of a larger theory of anisotropic pseudo-differential operators. 


\section{REFERENCES}

[1] Á. Bényi and M. Bownik, Anisotropic classes of homogeneous pseudodifferential symbols, Studia Math. 200 (2010), 41-66.

[2] M. Bownik, Anisotropic Hardy spaces and wavelets, Mem. Amer. Math. Soc. 164 (2003), no. 781, 122pp.

[3] M. Bownik, Atomic and molecular decompositions of anisotropic Besov spaces, Math. Z. 250 (2005) 539-571.

[4] M. Bownik and K.-P. Ho, Atomic and molecular decompositions of anisotropic Triebel-Lizorkin spaces, Trans. Amer. Math. Soc. 358 (2006), 1469-1510.

[5] R. R. Coifman and Y. Meyer, Au delá des opérateurs pseudo-différentiels, Astérisque $\mathbf{5 7}$ (1978).

[6] A.P. Calderón and R. Vaillancourt, A class of bounded pseudo-differential operators, Proc. Nat. Acad. Sci. U.S.A. 69 (1972), 1185-1187.

[7] H. O. Cordes, On compactness of commutators of multiplications and convolutions, and boundedness of pseudodifferential operators, J. Funct. Anal. 18 (1975), 115-131.

[8] C. Fefferman, $L^{p}$ bounds for pseudo-differential operators, Israel J. Math. 14 (1973), 413-417.

[9] G. Garello, Anisotropic pseudodifferential operators of type 1,1, Ann. Mat. Pura Appl. IV (1998), 135-160.

[10] L. Grafakos and R.H. Torres, Pseudodifferential operators with homogeneous symbols, Michigan Math. J. 46 (1999), 261-269.

[11] L. Hörmander, On the $L^{2}$ continuity of pseudo-differential operators, Comm. Pure Appl. Math. 24 (1971), 529-535.

[12] J.L. Journé, Calderón-Zygmund operators, pseudo-differential operators and the Cauchy integral of Calderón, Lecture Notes in Mathematics 994, Springer-Verlag (1983).

[13] H. Kumano-go, A problem of Nirenberg on pseudo-differential operators, Comm. Pure Appl. Math. 23 (1970), 115-121.

[14] H.-G. Leopold, Boundedness of anisotropic pseudo-differential operators in function spaces of Besov-Hardy-Sobolev type, Z. Anal. Anwendungen 5 (1986), 409-417.

[15] A. Miyachi, Estimates for pseudodifferential operators with exotic symbols, J. Fac. Sci. Univ. Tokyo Sect. IA Math. 34 (1987), 81-110.

[16] E.M. Stein, Harmonic analysis: real-variable methods, orthogonality, and oscillatory integrals, Princeton Univ. Press (1993).

Árpád Bényi, Department of Mathematics, 516 High Street, Western Washington

UNIVERSITY, BELLINGHAM, WA 98225-9063, USA

E-mail address: arpad.benyi@wwu.edu

Marcin Bownik, Department of Mathematics, University of Oregon, Eugene, or 97403-1222, USA

E-mail address: mbownik@uoregon.edu 